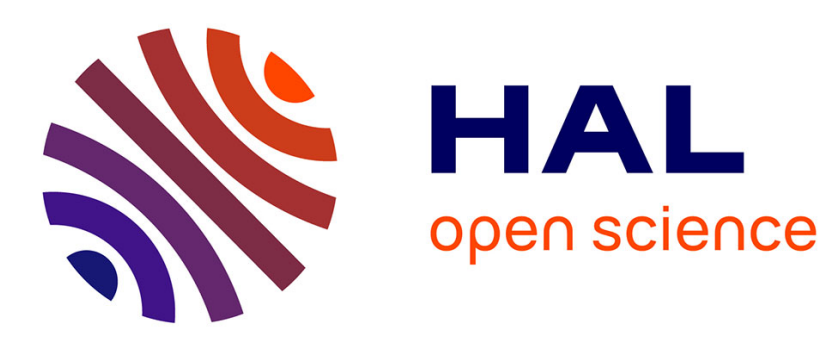

\title{
VALIRACUST, PARC ACOUSTIQUE POUR UNE RIVIÈRE
}

\author{
F. Daumal, H. Sanclemente, C. Castaño, R. Melendez, S. Tito
}

\section{To cite this version:}

F. Daumal, H. Sanclemente, C. Castaño, R. Melendez, S. Tito. VALIRACUST, PARC ACOUSTIQUE POUR UNE RIVIÈRE. Journal de Physique IV Proceedings, 1992, 02 (C1), pp.C1-163-C1166. 10.1051/jp4:1992132 . jpa-00251203

\section{HAL Id: jpa-00251203 https://hal.science/jpa-00251203}

Submitted on 1 Jan 1992

HAL is a multi-disciplinary open access archive for the deposit and dissemination of scientific research documents, whether they are published or not. The documents may come from teaching and research institutions in France or abroad, or from public or private research centers.
L'archive ouverte pluridisciplinaire HAL, est destinée au dépôt et à la diffusion de documents scientifiques de niveau recherche, publiés ou non, émanant des établissements d'enseignement et de recherche français ou étrangers, des laboratoires publics ou privés. 


\title{
VALIRACUST, PARC ACOUSTIQUE POUR UNE RIVIERE
}

\author{
F. DAUMAL, H. SANClEMENTE, C. CASTAÑO, R. MELENDEZ et S. TITO \\ Escola Tècnica Superior d'Arquitectura de Barcelona, av. Diagonal 649, SP-08028 Barcelona, Spain
}

\begin{abstract}
Within the framework of Acoustic Poetics and Acoustic Design, the project of the Acoustic Park "VALIRACUST" for the river Valira in Andorra provides an artistic and cultural place of recreation in the river area from Escaldes-Engordany and Andorra la Vella to santa Coloma.

In order to carry out this project, we intend to use differential sound sources; textures of the river bed (Smooth surfaces, stones, gravel, etc.), surface textures (waterfalls of different heights, springs, water sculptures, resonances, etc.), textures of facilities (recreation areas, pavements, outdoor furniture, sound games and other elements with the participation of water and sound).
\end{abstract}

\section{Résumé}

Dans le cadre de la Poétique Acoustique et la Conception Acoustique, le projet du Parc Acoustique "VALIRACUST" pour la rivière Valira d'Andorra, est presenté comme l'espace ludique-artistique et cultural qui, configurant un grand parc acoustique-aquifer, on peut disposer à travers la rivière des d'Escaldes Engordany et Andorra la Vella jusqu'à Santa Coloma.

Pour la réalisation de ce projet, nous avons l'intention d'utiliser des sources de sons differentiels; textures du fond de la rivière (surfaces lisses, pierres, graviers,..), avec des textures de surface (chutes d'eau de differentes hauteurs, fontaines, sculptures avec l'eau, résonances,...), textures d'équipement (zones de récréation, étude des pavés, mobilier urbain, jeux sonors, et d'autres éléments avec la participation de l'eau et du son).

\section{VALIRACUST}

La rapide et grande croissance des communes d'Escaldes-Engordany, Andorra la Vella et Santa Coloma au bord de la rivière Valira, ont provoqué la nécesité croissante de réserves d'espaces verds, mais souvent, ceux qui ont pu être crées sont restés séparés les uns des autres, du à ce que le tissu urbain a difficilement permit leur continuité.

Ainsi nous nous sommes intéressé à la proposition d'un parc acoustique de la rivière Valira "Valiracust" comme un espace ludico-acoustique et culturel qui rende possible la connexion entre ces trois communes. 
Le parc Valiracust a des intentions Artistiques parcequ'il se base sur des conceptions de design acoustiques avec des contenus poétiques. Culturelles, parceque chaque intervention acoustique doit être justement informée pour ce qui est de ses principes physiques, applications architectoniques, paysagistes, etc... De cette façon on désire que le visiteur du parc se trouve comme s'il été dans un musée de la science acoustique. Et finalement Ludiques: parceque grâce à des jeux acoustiques on peut intéresser les enfants, les adultes ou les plus vieux, optenant un lieu de divertissements singulier et unique.

Ce projet a été réalisé par l'Atelier d'Architecture Acoustique de I'Ecole Technique supérieure d'Architecture de Barcelone.

\section{LA RIVIERE COMME UN COMPOSANT URBAIN, ANALISE GENERALE.}

En faisant un cheminement trés général, obserbant la rivière et son intégration dans la ville, on se rend compte qu'il existe une grande séparation entre les deux. PIus avant, nous pouvons dire que la rivière a été considéré comme une canalisation des résidus liquides et non pas comme un composant urbain. Depuis son arrivée à la ville, la rivière se trouve etoufée par les batiments qui l'absorbent et privent de toutes vues. A tout ceçi, il faut ajouter aussi une forte topographie.

\section{ZONIFICATION, ANALISE ET PROPOSITIONS SPECIFIQUES.}

Le "Leitmotiv" du projet c'est que le jeu avec les sons, peut établir la differentiation des zones du parc et donner les acoustiques charactéristiques à des points concrets. Pour celà, on a étudié le parc valiracust suivant les types les plus importants du cheminement de la rivière. On compte neuf différentes zones.

Zone 1: Residentielle, où la hauteur des constructions de logements a etouffé la riviere qui s'est convertie en la partie postérieure des bâtiments, sans aucun intérêt. Nous proposons d'ouvrir des zones complémentaires sur les berges de la rivière afin de contrecarrer la haute densité de la zone.

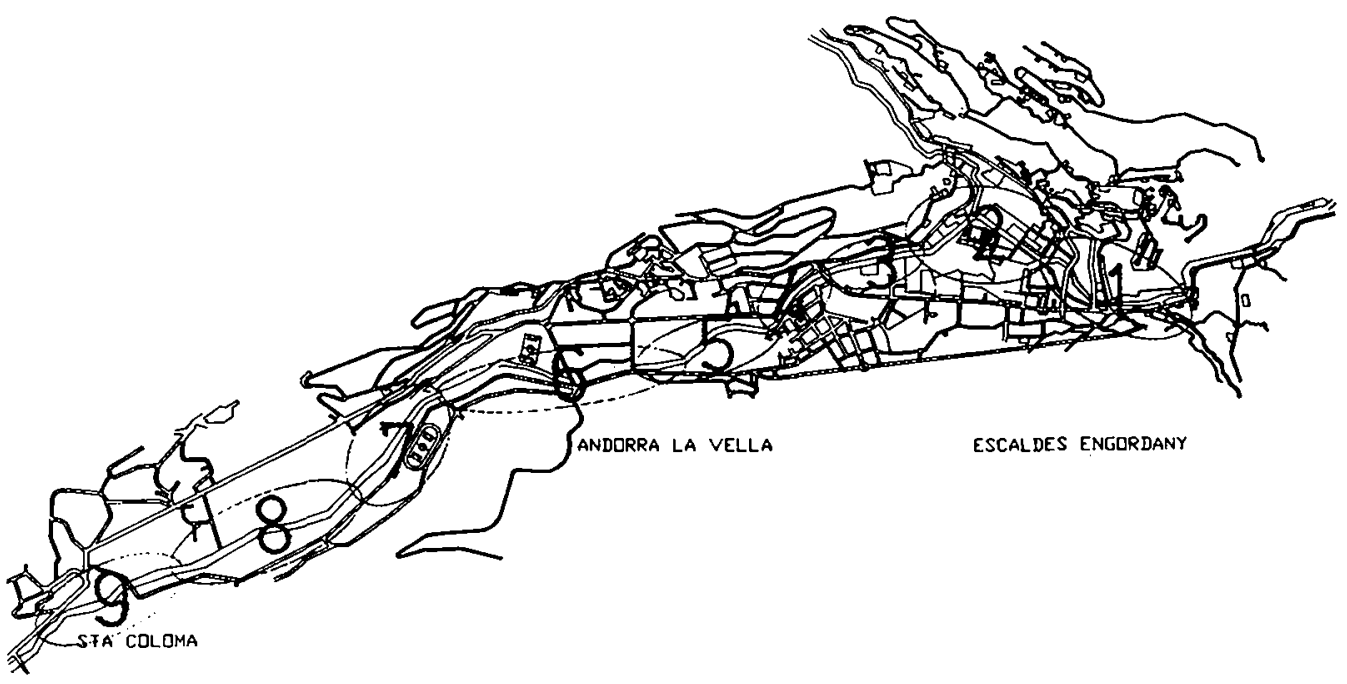


Au niveau acoustique, nous jouons avec le son afin d'établir une différentiation des zones et donner des acoustiques charactéristiques à des points concrets. Pour cette réalisation, on combine des "Textures du fond" de la rivière (surfaces lisses, pierres, graviers,..) cascades d'eau à différentes hauteurs, fontaines, sculptures acoustiques avec l'eau, résonances, etc...



Au niveau paysagiste, nous recherchons des effets visuels afin de gagner 1 'attention des gens grace à des miroirs d'eau (eau casi dormante ou movements rapides de l'eau, essayant de suppimer l'impact froid et séparateur du mur de soutainement en utilisant le maximum d'éléments naturels, propres à la rivière.

Zone 2: Récréative. La rivière içi est plus accessible car on n'a pas encore construit le mur de soutainement qui la sépare de la ville dans presque tout son cheminement. Celà permet aussi de profiter d'un contacte visuel avec le paysage et de perspectives plus amples. Nous proposons l'inégration de manière directe en amenant l'eau jusqu'aux zones des enfants, de repos des adultes et des espaces des jeunes. Ce contacte directe fera que les gens réavivent ce rôle actif que la rivière doit présenté à la ville.

Pour celà, on procurera la conduite d'eaux thermales pour faciliter l'accès, des travaux de sculptures avec eau, jeux et autres éléments avec la participation de I'eau. Afin d'accomplir cet objectif, il est important la jonction de la zone récéative avec la ville. Ceci peut se faire au moyen d'une symbolique urbaine facilment identifiable et lue, comme peuvent être des pavimentations spéciales sonores, bancs de repos, sculptures sonores, zones de jeux et autres éléments. 
Zone 3: Commerciale. L'effet paysagiste redevient froid, du aux murs de soutainements de la rivière et au manque de considérations des éléments urbanistiques.

Nous proposons de créer des lieux de repos appuyés à la rivière, en les pavimentant en conséquences et en les renforçant avec du mobilier urbain, comme des bancs et luminaires, transformant ces percées des commerces en fugues vers la rivière.

Zone 4: Commerciale-Residentielle. Il n'existe pas de relation directe et facile avec la rivière de par la forte présence des murs de soutainements et l'abandon du nettoyage du fond de la rivière. Il faut trouver l'ouverture visuelle de la riviere, maintenant la peu qui existe actuellement. Pour celà, on doit empécher la construction sur les zones annexes de la rivière et promouvoir la création d'espaces verds acoustiques sus ses rives, à l'aide d'arbres sonores.

Zone 5: Récréative. La rivière apparait libre de murs de soutainements, laissant des marges naturelles, les vues ne sont pas coupees et on peut accéder directement à l'eau. Nous proposons un travail de maintenance et consolidation de la zone, créant des jeux d'eau acoustiques, étangs avec eaux régulables qui permettent la présence de canards, création d'une plage facilitant la baignade des gens en été, protection du cour de la rivière à partir de gradins acoustiques qui remplacent la fonction des murs de soutainement existant dans les autres zones, etc...

Zone 6: Résidentielle-Récréative. On peut obtenir une milleure intégration des espaces sportifs et zones de logements existants avec les autres zones, ainsi que canaliser et reconduir les eaux sanitaires qui içi échouent directement à la rivière. On peut aussi empécher la privatisation des rives de la riviere, créant au minimum une zone de promenade publique acoustique parrallèle à la rivière.

Zone 7 Récréative. Heureusement, les rives de la rivière encore conservent les arbres et la végétation naturelle. On prévoit la conexion de cet equipement avec la rivière gràce à des promenades et circuits de joggings, complémentés de quelques sculptures sonores aériennes.

Zone 8: Agricole. De nouveau, la rivière circule limitée dans des murs gui protegent les champs des inondations. Ceux-çi convertissent la rivière en un canal d'irrigation. Nous proposons de créer une politique urbanistique qui réglemente le développement urbain de cette zone, la consolidant comme terrain rural ou urbain mais avec des exigences d'interaction rivière-vilie, et la création du champ sonore.

Zone 9: Résidentielle-Récréative. Nous prévoyons comme une activité prioritaire, stabiliser et promouvoir en tant que zone récréativerésidentielle. Le traitement des eaux de la rivière est prioritaire afin de développer le rappochement des gens avec la rivière, et ainsi d'offrir des activités acoustiques variées, tant actives comme passives.

\section{CONCLUSION}

Avec le parc Valiracust, il se prévoit pour la première fois une intervention le long de toute une rivière afin de récupérer sa poetique et la transformant en une mélodie et un cheminement acoustique, consistant à ce qu'elle devienne le point d'union entre ces trois villes par lesquelles elle passe.

Ceçi doit comporter un changement trés positif et une nouvelle image d'une ville préoccupée par son paysage, ses habitants et ses visiteurs. 